\title{
Effectiveness of Mobile Health Augmented Cardiac Rehabilitation on Behavioural Outcomes among Post-acute Coronary Syndrome Patients: A Randomised Controlled Trial
}

\author{
Saima Manzoor ${ }^{1}$, Aliya Hisam¹, Sohail Aziz², Syed Fawad Mashhadi ${ }^{1}$ and Zia Ul Haq ${ }^{3}$ \\ ${ }^{1}$ Department of Community Medicine, Army Medical College, National University of Medical Sciences, Rawalpindi, Pakistan \\ ${ }^{2}$ Department of Interventional Cardiology, Armed Forces Institute of Cardiology and National Institute of Heart Diseases (AFIC/NIHD), \\ Rawalpindi, Pakistan \\ ${ }^{3}$ Department of Public Health \& Social Sciences, Khyber Medical University, Peshawar, Pakistan
}

\begin{abstract}
Objective: To determine the effectiveness of mobile health augmented cardiac rehabilitation (MCard) on behavioural factors among post-acute coronary syndrome (post-ACS) patients.

Study Design: Randomised controlled trial.

Place and Duration of Study: Armed Forces Institute of Cardiology (AFIC), from January 2019 till March 2021.

Methodology: Post-ACS patients were assigned to one of two groups: intervention (counselling, brief text messages, and standard post-ACS care) or control (no intervention) (standard post-ACS care). Healthy eating (Healthy eating assessment tool) and physical activity (IPAQ tool), medication compliance, smoking, salt intake, blood pressure (BP), and weight self-monitoring were assessed as behavioural factors. Data were collected three times for a six-month follow-up period (baseline, 12 and 24 weeks). Result: At 12 weeks, 121 (76.62\%) of the 160 patients enrolled patients (80 in each group) were analysed; and at 24 weeks, $119(74.38 \%)$ were analysed. Mean MET score at baseline was 1170 control vs. 1161 intervention $(p=0.940)$, at 12 weeks 826 vs. $934(p=0.390)$; and at 24 weeks was 925 vs. $1454(p=0.007)$. Healthy eating value at baseline was 36.43 control vs. 36.38 intervention $(p=0.897)$, at 12 weeks 38.76 vs. $40.98(p<0.001)$; and at 24 weeks 40.12 vs. $43.54(p<0.001)$. There was also a significant difference in salt intake $(p=0.008)$ and healthy diet $(p=0.012)$, but not in medicine compliance, smoking behaviour, self-monitoring of BP and weight.

Conclusion: The MCard positively impacts the post-ACS participants' behaviours in terms of physical activity, healthy eating, and salt restriction. MCard evidenced as a feasible intervention in terms of having lasting behaviour modification among this vulnerable patient population.
\end{abstract}

Key Words: Acute coronary syndrome, Myocardial infarction, Cardiovascular diseases, Cardiac rehabilitation, Healthy diet, Physical activity, Smoking, Tobacco, Telemedicine, Lifestyle modification.

How to cite this article: Manzoor S, Hisam A, Aziz S, Mashhadi SF, Haq ZU. Effectiveness of Mobile Health Augmented Cardiac Rehabilitation on Behavioural Outcomes among Post-acute Coronary Syndrome Patients: A Randomised Controlled Trial. J Coll Physicians Surg Pak 2021; 31(10):1148-1153.

\section{INTRODUCTION}

Acute coronary syndrome (ACS) includes acute ST-elevation myocardial infarction, unstable angina, and non-ST-segment elevation and is one of the most common causes of premature deaths. ${ }^{1}$

Correspondence to: Dr. Aliya Hisam, Department of Community Medicine, Army Medical College, National University of Medical Sciences, Rawalpindi, Pakistan E-mail: aaleya@yahoo.com

Received: May 26, 2021; Revised: September 01, 2021; Accepted: September 17, 2021

DOI: https://doi.org/10.29271/jcpsp.2021.10.1148
Risk factors of ACS are both non-modifiable (age, gender, family history, ethnicity) and modifiable. The modifiable factors that need to be focused on are smoking, including first, second and third-hand smoking, an unhealthy diet, which leads to overweight, obesity, increased glycemic index, uncontrolled lipid profile, high blood pressure (BP) related to high salt intake, and sedentary lifestyle. ${ }^{2}$

Physical inactivity is a significant contributor to heart diseases. Adults with low physical activity are at increased cardiometabolic risk. Being physically active, helps reduce weight, lower BP, which helps minimise the risk of developing ACS. ${ }^{3}$ Diet is likely to play a part in the risk of a cardiac adverse event. Consumption of non-processed fruits and vegetables, whole grains, and lean protein is encouraged in healthy dietary habits; 
whereas, sugar-sweetened products are restricted. ${ }^{4}$ Smoking is known to be the underlying trigger for myocardial infarction. Smoking primary prevention and quitting is a critical factor in the prevention and relapse of ACS, and all these modifiable risk factor preventions depend upon CR and its utilisation. ${ }^{5}$

Realising that sociodemographic factors, unhealthy dietary habits and lack of physical activity are associated with increased risk of cardiac events, a need arises to reduce these risk factors. ${ }^{6}$ Lifestyle modification are very beneficial in controlling the increasing burden of ACS, as it has shown that lifestyle behaviour modifications can alter ACS course. ${ }^{7}$ Cardiac rehabilitation (CR) is a pillar of secondary prevention following the ACS event. ${ }^{8} \mathrm{CR}$ incorporates graduated cardiovascular exercise, risk factor modification, education, and social support service, which improve behaviours. ${ }^{9}$ A study in Pakistan showed that $C R$, following myocardial infarction, effectively improved health-related quality of life; and shall be implemented at healthcare institutions in Pakistan. ${ }^{10}$ Although the CR has demonstrated effectiveness in improving patient's behaviours, participation in these programmes is low. ${ }^{11}$ Mobile health (mhealth) interventions are being incorporated in CR programmes to increase the utilisation of CR programme and a favourable prognosis. ${ }^{12}$ Telephonic counselling for behavioural and clinical factors in post-ACS patients is believed to reduce mortality and morbidity in developed countries, but this aspect is unexplored in developing countries.

There is a paucity of studies on the effectiveness of mHealth incorporated CR in improving post-ACS patients' behaviours.

This study aimed to find out the effectiveness of MCard on the behaviour of post-ACS patients, who were admitted to a tertiary care hospital through a randomised controlled trial.

\section{METHODOLOGY}

A two-arm randomised controlled trial was conducted at Armed Forces Institute of Cardiology; and National Institute of Heart Diseases (AFIC-NIHD), a tertiary care cardiac centre in Rawalpindi, Pakistan, from January 2019 till March 2021. The trial is registered with the Australian New Zealand Clinical trial registry (ANZCTR) under trial registration No: ACTRN12619 $001731189^{13}$ and its detailed protocol have been published in the Pakistan Journal of Medical Sciences. ${ }^{14}$

Study subjects included all ACS patients (ST-Elevation Myocardial Infarction, Non-ST-elevation Myocardial Infarction, and Unstable Angina) in the last month. Patients fulfilling eligibility criteria were randomly allocated to control or intervention groups by a computerised random number generator. In addition to standard post-ACS treatment, the intervention group received the MCard intervention, a medically monitored cardiac rehabilitation programme. During the hospital stay, the first phase of the MCard included individualised counselling. The second phase included diurnal mobile texting of standardised messages about healthy lifestyle changes, using a specially created app. Before participating in the study, all participants were asked to provide written informed consent. Data were collected at baseline 12 weeks follow-up and at 24 weeks follow-up.

Along with demographic details, physical activity level and dietary habits were assessed through the international physical activity questionnaire (IPAQ) and healthy eating assessment questionnaire (HEQ). The IPAQ scoring was that those having less than 600 were in a low category, those having 600-3000 MET-minutes were classified as moderate; whereas, having more than 3000 were of high category group. ${ }^{15}$ Dietary assessment scores were calculated by using HEQ. Diet scores were calculated by the summation of intake of fruits, vegetables, fried foods, snacks, sugar, milk products, and meat as reported by the participants. HEQ comprises ten items and scoring ranges from values of 10-50; categories are fair (20-29), good (30-39), and excellent (40-50). ${ }^{16}$ Smoking status was assessed with some basic questions using a self-constructed questionnaire and classified into non-smokers, currentsmokers, and ex-smokers. Moreover, a measure of the number of cigarettes smoked per day was considered. Questions related to salt reduction to less than a teaspoon per day, walk for at least 20 minutes, thrice-weekly and taking medication according to doctor'sprescription weresubjectively assessed. Subsequently, checking BP and weight at least once weekly and the subjective preference for a healthy diet were assessed.

STATA version 14 was used to enter and analyse data. The categorical data were presented using frequencies and percentages, and the two groups were compared using Chi-square test. The continuous data of the two groups were presented as a mean, with a standard deviation; and an independent-sample t-test was applied to find an association of MCard and participants' behaviours of both the groups. A p-value of less than 0.05 was used to determine the level of significance.

\section{RESULTS}

A total of 160 post-ACS patients were enrolled. They were randomised in a 1:1 ratio into groups of 80 in each (control and intervention). The mortality was 22 (13.8\%); whereas, 19 (11.9\%) were lost to follow-up. Thus 119 (74.4\%) participants completed six months of assessment. The mean age was $52.67 \pm 8.47$ years, with no significant difference between the two groups $(p=0.124)$. The majority were males ( $n=126,78.80 \%)$, Punjabis $(119,74.4 \%)$, and about half of the population had no formal education (49, $30.6 \%)$.

According to IPAQ scoring, at 12 weeks follow-up, most of the intervention group participants were in the moderate group, 50 (70.42\%); and at 24 weeks follow-up, 53 (75.71\%) participants. At 12 and 24 weeks, the control group was also in the moderate group, $32(64.00 \%)$ and 32 (65.31\%), respectively. There was no significant difference in physical activity levels between the two study groups at baseline and 12 weeks follow up, as observed by the mean MET minutes (control: 826.77, p-value $=0.390$ ); whereas, the mean MET min was significantly different among the control and intervention groups at 24 weeks follow-up; (control: 925.64, 95\% Cl: $724.58,1126.70$ vs. intervention: $1454.50,95 \% \mathrm{Cl}$ : 1163.84, 1745.15, $\mathrm{p}=0.007$ ), respectively (Table I and Figure 1a). 
Table I: Behavioral outcomes assessment using IPAQ and HEQ scoring of MCard participants.

\begin{tabular}{|c|c|c|c|c|c|c|c|c|}
\hline & & & $\begin{array}{c}\text { Baseline } \\
\text { n (\%) }\end{array}$ & p-value & $\begin{array}{c}12 \text { weeks follow-up } \\
n(\%)\end{array}$ & p-value & $\begin{array}{c}24 \text { weeks follow-up } \\
\text { n (\%) }\end{array}$ & p-value \\
\hline \multicolumn{9}{|c|}{ International physical activity questionnaire scoring of MCard participants } \\
\hline \multirow{6}{*}{ IPAQ Scoring } & \multirow{2}{*}{ Low } & Control & $18(22.50)$ & \multirow{6}{*}{0.933} & $18(36.00)$ & \multirow{6}{*}{0.483} & $16(32.65)$ & \multirow{6}{*}{0.010} \\
\hline & & Intervention & $20(25.00)$ & & $20(28.17)$ & & $9(12.86)$ & \\
\hline & \multirow{2}{*}{ Moderate } & Control & $60(75.00)$ & & $32(64.00)$ & & $32(65.31)$ & \\
\hline & & Intervention & $58(72.50)$ & & $50(70.42)$ & & $53(75.71)$ & \\
\hline & \multirow{2}{*}{ High } & Control & $02(2.50)$ & & - & & $1(2.04)$ & \\
\hline & & Intervention & $02(2.50)$ & & $1(1.41)$ & & $8(11.43)$ & \\
\hline \multicolumn{9}{|c|}{ A healthy eating score of the MCard participants } \\
\hline \multirow{6}{*}{ HEQ Score } & \multirow{2}{*}{ Fair } & Control & - & \multirow{6}{*}{0.265} & $2(4.00)$ & \multirow{6}{*}{0.042} & - & \multirow{6}{*}{$<0.001$} \\
\hline & & Intervention & $2(2.50)$ & & - & & - & \\
\hline & \multirow{2}{*}{ Good } & Control & $72(90.00)$ & & $23(46.00)$ & & $20(40.82)$ & \\
\hline & & Intervention & $67(83.75)$ & & $22(30.99)$ & & $06(08.57)$ & \\
\hline & \multirow{2}{*}{ Excellent } & Control & $8(10.00)$ & & $25(50.00)$ & & $29(59.18)$ & \\
\hline & & Intervention & $11(13.75)$ & & $49(69.01)$ & & $64(91.43)$ & \\
\hline
\end{tabular}

Table II: Behavioral outcomes among the control and intervention groups at baseline and follow-ups.

\begin{tabular}{|c|c|c|c|c|c|c|c|c|c|}
\hline & \multicolumn{3}{|l|}{ Baseline } & \multicolumn{3}{|c|}{12 weeks follow-up } & \multicolumn{3}{|c|}{24 weeks follow-up } \\
\hline & Yes & No & & Yes & No & & Yes & No & \\
\hline & n (\%) & n (\%) & p-value & n (\%) & n (\%) & p-value & $\mathbf{n} \%$ & n (\%) & $\mathrm{p}$-value \\
\hline \multicolumn{10}{|c|}{ Medicine's compliance } \\
\hline Control & $57(71.25)$ & $23(28.75)$ & \multirow{2}{*}{0.472} & $48(94.12)$ & $03(5.88)$ & \multirow{2}{*}{0.400} & $48(97.96)$ & $01(2.04)$ & \multirow{2}{*}{0.798} \\
\hline Intervention & $61(76.25)$ & $19(23.75)$ & & $69(97.18)$ & $02(2.82)$ & & $69(98.57)$ & $01(1.43)$ & \\
\hline \multicolumn{10}{|c|}{ Salt restriction } \\
\hline Control & $44(55.00)$ & $36(45.00)$ & \multirow{2}{*}{0.057} & $37(74.00)$ & $13(26.00)$ & \multirow{2}{*}{0.311} & $37(75.51)$ & $12(24.49)$ & \multirow{2}{*}{0.008} \\
\hline Intervention & $32(40.00)$ & $48(60.00)$ & & $58(81.69)$ & $13(18.31)$ & & $65(92.86)$ & $5(7.14)$ & \\
\hline \multicolumn{10}{|c|}{ Healthy diet preference } \\
\hline Control & $65(81.25)$ & $15(18.75)$ & \multirow{2}{*}{0.527} & $39(78.00)$ & $11(22.00)$ & \multirow{2}{*}{0.007} & $40(81.63)$ & 09 (18.37) & \multirow{2}{*}{0.012} \\
\hline Intervention & $68(85.00)$ & $12(15.00)$ & & $67(94.37)$ & $04(5.63)$ & & $67(95.7)$ & $03(4.25)$ & \\
\hline \multicolumn{10}{|l|}{ Check BP } \\
\hline Control & $49(61.25)$ & $31(38.75)$ & \multirow{2}{*}{0.320} & $47(94.00)$ & $03(6.00)$ & \multirow{2}{*}{0.820} & $47(95.92)$ & $02(4.08)$ & \\
\hline Intervention & $55(68.75)$ & $25(31.25)$ & & $66(93.00)$ & $05(7.00)$ & & $67(95.71)$ & $03(4.29)$ & \\
\hline \multicolumn{10}{|c|}{ Check weight } \\
\hline Control & $34(42.50)$ & $46(57.50)$ & \multirow{2}{*}{0.100} & $40(78.43)$ & $11(21.57)$ & \multirow{2}{*}{0.191} & $39(79.59)$ & 0.956 & \multirow{2}{*}{0.268} \\
\hline Intervention & $24(30.00)$ & $56(70.00)$ & & $62(87.32)$ & $9(12.68)$ & & $61(87.14)$ & $09(12.86)$ & \\
\hline \multicolumn{10}{|l|}{ Walk } \\
\hline Control & $51(63.75)$ & $29(36.25)$ & \multirow{2}{*}{0.418} & $40(80.00)$ & $10(20.00)$ & \multirow{2}{*}{0.880} & $37(75.51)$ & $12(24.49)$ & \multirow{2}{*}{0.008} \\
\hline Intervention & $46(57.50)$ & $34(42.50)$ & & $56(78.87)$ & $15(21.13)$ & & $65(92.86)$ & $05(7.14)$ & \\
\hline Non-smoker & \multicolumn{2}{|l|}{ n (\%) } & & \multicolumn{2}{|l|}{ n (\%) } & & \multicolumn{2}{|l|}{ n (\%) } & \\
\hline Control & $51(63.75)$ & & & $27(54.00)$ & & & $26(53.06)$ & & \\
\hline Intervention & $52(65.00)$ & & & $44(61.97)$ & & & $45(64.29)$ & & \\
\hline Current smol & & & & & & & & & \\
\hline Control & $14(17.50)$ & & & $7(14.00)$ & & & 7 (14.29) & & \\
\hline Intervention & $16(20.00)$ & & 0.788 & $11(15.49)$ & & 0.508 & $9(12.86)$ & & 0.432 \\
\hline Ex-smoker & & & & & & & & & \\
\hline Control & $15(18.75)$ & & & $16(32.00)$ & & & $16(32.65)$ & & \\
\hline Intervention & $12(15.00)$ & & & $16(22.54)$ & & & $16(22.86)$ & & \\
\hline
\end{tabular}

The majority of the intervention group at 24 weeks follow-up were significantly more in the excellent category as healthy eating was analysed $(n=64,91.4 \%, p<.001)$. There was an improvement in the dietary habits as shown by the mean HEQ value among intervention group from baseline to 12 and 24 weeks follow-up; (control: $36.43,95 \% \mathrm{Cl}: 35.93$, 36.92, vs. intervention: $36.38,95 \% \mathrm{Cl}: 35.78,36.97, \mathrm{p}$ $=0.897$ ); at 12 weeks (control: $38.76,95 \% \mathrm{Cl}: 37.75,39.76$ vs. intervention: $40.98,95 \% \mathrm{Cl}: 40.31,41.65, \mathrm{p}<0.001$ ) and at 24 weeks (control: $40.12,(95 \% \mathrm{Cl}: 39.15,41.08$, vs. intervention: $3.5,95 \% \mathrm{Cl}: 42.83,44.25, \mathrm{p}<0.001$, (Table I and Figure $1 b)$.

The subjective assessment of healthy diet preferences showed a marked improvement at 12 and 24 weeks followup; at 12 weeks, control: $39(/ 78 \%)$ vs. intervention: $67(94.3 \%)(p=0.007)$ and at 24 weeks, control: $40(81.6 \%)$ vs. intervention: $67(95.7 \%)(p=0.012)$. Of the 160 partici- pants, $30(18.75 \%)$ were regular smokers. The smoking behaviour of both groups was not affected significantly by the intervention. However, an assessment of the smoking behaviour indicated that smoking cessation dropped by $8.8 \%$. The subjective medicine compliance also improved from baseline to 12 weeks and 24 weeks follow up; at baseline control: 57 (71.25\%) vs. intervention: 61 (76.25\%, $p=0.472)$, at 12 weeks, control: $48(94.12 \%) v s$. intervention: $69(97.18 \%, p=0.400)$ and at 24 weeks, control: 48 (97.96\%) vs. intervention: 69 (98.57\%, $p=0.798)$. The participants in the intervention group showed a better adoption of salt restriction in their diet towards the end of the trial at 24 weeks though it was not significant at 12 weeks: control: 37 (74\%) vs. intervention: $58(81.69 \%, p=0.311)$ whereas at 24 weeks, salt restriction in controls: $37(75.5 \%)$ vs. intervention: $65(92.8 \%, p=0.008)$. 


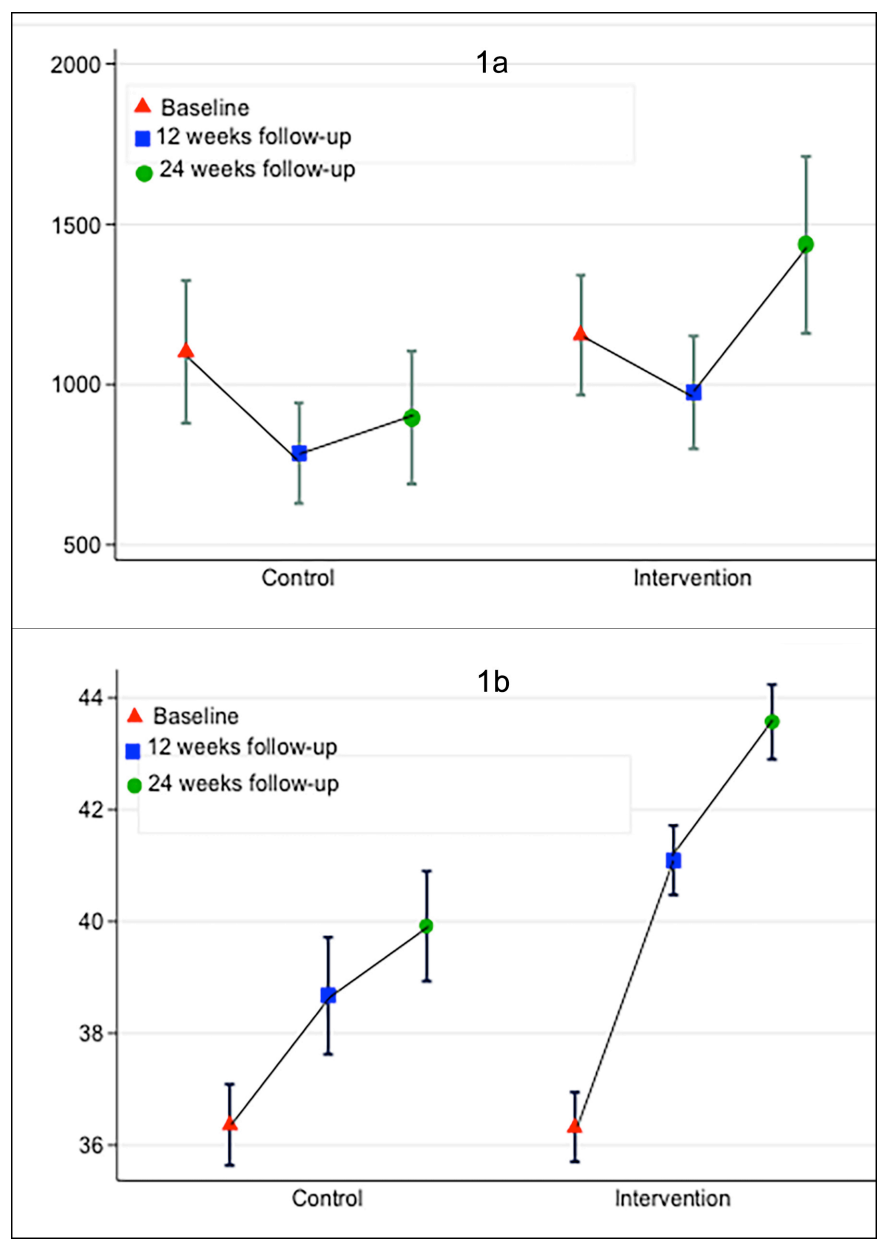

Figure $1(a, b)$ : International physical activity assessment and healthy eating assessment among the post-ACS at baseline, 12 weeks and 24 weeks.

Self-monitoring, including checking of blood pressure (BP) and weight, was also practised by the participants at least once weekly; at 12 weeks checking BP in control was 47 $(94 \%)$ and in intervention $66(93 \%)(p=0.820)$, at 24 weeks in control: $47(95.2 \%)$ vs. intervention: $67(95,7 \%, p=0.956)$. Similarly checking weight at 12 weeks, control: 40 (78.4\%) vs. intervention: 62 (87.3\%, $\mathrm{p}=0.191)$; at 24 weeks control: $39(79.5 \%)$ and intervention: $61(87.1 \%, p=0.268)$. Walking for 20 minutes at least thrice a week showed no significant improvement at 12 weeks; where, among control were 40 $(80 \%)$ and among intervention were $56(78.8 \%, p=0.880)$ but was markedly better at 24 weeks, control: 37 (75.5\%) and intervention: 65 (92.8\%, $p=0.008$, Table II).

\section{DISCUSSION}

MCard is the first novel approach that is flexible, affordable, and appealing to change behavioural outcomes (physical activity, healthy diet, and salt restriction) by increasing awareness and incorporating self-care practices among postAS patients. This 24-weeks follow-up trial provided evidencebased medicine regarding the effectiveness of secondary prevention programmes, MCard, on post-ACS patients behavioural parameters (physical activity, dietary intake, and smoking status). MCard is associated with improvement in post-ACS patient's behavioural outcomes.

Reid et al. conducted a study on 137 post-ACS patients. He compared a mHealth CR (CardioFit) to standard care, concluding that physical activity at 24 weeks was subjectively increased in the intervention group. ${ }^{17}$ Exercise-based CR also achieves clinically significant changes in physical strength and physical functioning in ACS patients, according to a meta-analysis. ${ }^{18}$ All these studies favour this study and concluded that MCard could improve physical activity among post-ACS patients, which is undoubtedly one of the modifiable risk factors to be targeted. However, on the contrary, one meta-analysis concluded that there was a minimal improvement from exercise in a mHealth based $\mathrm{CR}{ }^{19}$

Diet counselling increased adherence to proper diet, as shown by the increase in HEQ scores at 12 and 24 weeks follow-up. Subjective assessment of healthy diet preference also showed marked improvement. Zhong et al., in their study, found that eating fruits, vegetables, whole grains, fish, nuts, legumes, low- or no-fat dairy products, and some vegetable oils decreased the risk of cardiovascular disease and mortality. ${ }^{20}$ Quitting smoking is hard, even after lifethreatening events such as ACS syndrome. This study revealed that smoking cessation dropped by $8.8 \%$ only, which was not statistically significant. A study in Italy revealed that in post-myocardial infarction, smokers' percentage fell by $23.5 \%(p=0.01)$, following a counselling intervention as CR program. ${ }^{21}$ Another study showed that smokers at a high-risk baseline might be more motivated to quit smoking, and thus more likely to respond to mHealth smoking cessation programmes. ${ }^{22}$

There were no difference in medical compliance between intervention and control groups. A similar study revealed that medication non-adherence by patients with ACS is linked to a lack of understanding and co-morbidity. ${ }^{23}$ Qualitative studies can be planned to understand the non-compliance among post-ACS patients. A study in Portugal revealed that after the adoption of salt reducing strategies, there was a population-wide reduction in high blood pressure and cardiovascular diseases. ${ }^{24}$ As significant adoption of salt restriction was observed in this trial (p-0.008), if followup of the same participants can be planned, insight into the $\mathrm{BP}$ reduction can be assessed. Another study involved patient education programmes in post ACS patients. In a study educating post-ACS patients at 45 and 180 days follow-up, subjects' body weight, BMI, and health status showed no improvement. ${ }^{25}$

The limitations of this study are that, it is a mono-centre study. If this was a multicentre trial, we could generalise study findings to all the post-ACS patients in Pakistan. Secondly, patients coming to this tertiary care centre might be more or less motivated, adherent or non-adherent to 
evidence-based guidelines, having chances of participation bias. More research integrating emerging technologies is needed in high-income and low-to-middle-income countries to evaluate the efficacy of affordable and feasible CR models. Studies on the cost-effectiveness of mHealth-enhanced $C R$ could be conducted in the future. MCard has also been shown to improve $C R$ attendance and self-management skills, although the long-term effects will need to be investigated in more extensive studies in the future. Rising MCard services across the country could help many postACS patients improve their physical and mental health, while also lowering their non-communicable disease burden.

MCard, a noteworthy intervention, can be adopted at the tertiary healthcare delivery level by training nurses and junior doctors, who can help patients acquire better health behaviours, prevent recurrence and decrease morbidity and mortality of this vulnerable group. Auxiliary health staff like lady health workers can also be employed at low cost to work in such programmes and trained to do counselling and follow-up on mobile phones. All of these patients were mobile phone owners, so this seems to be a very cost-effective CR model. Once established at the tertiary level, it can be implemented at primary and secondary healthcare levels, too.

\section{CONCLUSION}

The MCard positively impacts the behaviours of post-ACS participants, in terms of physical activity, healthy eating, and salt restriction. MCard evidenced as a feasible intervention in terms of having lasting behaviour modification among this vulnerable patient population. It did not significantly affect the participants' smoking behaviours, but the self-monitoring practices were improved more among the MCard group than the control group.

\section{DISCLOSURE:}

The authors disclose that this trial is a PhD in Public Health Project of Dr. Aliya Hisam, which is supervised by Prof. Zia UI Haq, Vice Chancellor, Khyber Medical University, and cosupervised by Prof. Sohail Aziz, Consultant Interventional Cardiologist, as site co-supervisor, AFIC-NIHD.

\section{ACKNOWLEDGMENT:}

The authors would like to thank the administration and medical staff at AFIC \& NIHD for their assistance and help in ensuring that the trial went smooth. Professors and public health experts from the Institute of Public Health and Social Sciences (IPH\&SS) at KMU in Peshawar and Army Medical College (AMC) at the National University of Medical Sciences (NUMS) in Rawalpindi, Pakistan, offered valuable advice and mentorship (no external funding was received for this project). We want to thank all of our co-workers and colleagues whose input smoothly helped us execute the MCard trial.

\section{ETHICAL APPROVAL:}

The clinical trial was authorised by the Khyber Medical University Ethical Review Committee (DIR/KMU-E$\mathrm{B} / \mathrm{MH} / 000486)$ and the Armed Forces Institute of Cardiology (AFIC-IERB-SOP-15).

\section{PATIENTS' CONSENT:}

Each participant signed a written informed consent form before enrolment.

\section{CONFLICT OF INTEREST:}

The authors declared no conflict of interest.

\section{AUTHORS' CONTRIBUTION:}

SM: Literature review, a writeup of the study protocol and discussion, designed the trial methodology, intervention execution, data acquisition and data analysis, drafting the manuscript, and submitting the revised version.

$\mathrm{AH}$ : Literature review, conceived the idea, designed the trial methodology, intervention execution, data analysis and writeup and editing of the manuscript. Final drafting for the journal and submitting revised version and enduring content accuracy and communication.

SA: Trial methodology concept, data acquisition, trial monitoring, accuracy, and integrity of results, critically revising the manuscript for final submission.

SFM, ZUH: Critical review of the trial idea and methodology, administrative and logistic support, planning of data analysis and statistical analysis, revised the manuscript for important intellectual content, and final editing of the manuscript for the final version to be submitted.

All authors are fully responsible for study integrity and result's accuracy.

\section{REFERENCES}

1. Yang $H$, Zuo $H$, Jia $S$, Ye $P$, Xing $H$, Zhao $X$, et al. Risk factor distribution features and trends of young adults with the first acute coronary syndrome. Zhonghua xin xue Guan Bing za zhi 2021; 49(3):242-49. doi: 10.3760/CMA j.cn11214820200417-00324.

2. Brown JC, Gerhardt TE, Kwon E. Risk factors for coronary artery disease 2020 [cited 2021]. Available from: http://www.ncbi.nlm.nih.gov/books/NBK554410/.

3. Moreira JB, Wohlwend M, Wisløff U. Exercise and cardiac health: Physiological and molecular insights. Nat Metab 2020; 2(9):829-39. doi.org/10.1038/s42255-020-0262-1.

4. Fischer NM, Pallazola VA, Xun H, Cainzos-Achirica M, Michos ED. The evolution of the heart-healthy diet for vascular health: A walk through time. Vasc Med 2020; 25(2):184-93. idoi.org/10.1177/1358863X19901287.

5. Goettler D, Wagner M, Faller H, Kotseva K, Wood D, Leyh R, et al. Factors associated with smoking cessation in patients with coronary heart disease: A cohort analysis of the German subset of EuroAspire IV survey. BMC Cardiovasc Disord 2020; 20:1-9. doi.org/10.1186/s12872-020-01429-w.

6. Sajid MR, Muhammad N, Zakaria R, Shahbaz A, Nauman A. Associated factors of cardiovascular diseases in Pakistan: 
Assessment of path analyses using warp partial least squares estimation. Pakistan J Stat Oper Res 2020; 265-77. doi.org/10.18187/pjsor.v16i2.3075.

7. Saeed S, Kanaya AM, Bennet L, Nilsson PM. Cardiovascular risk assessment in South and Middle-East Asians living in the Western countries. PakJ Med Sci 2020; 36(7):1719-25. doi: 10.12669/pjms.36.7.3292.

8. Lawless M, Harrison AS, Doherty P. Multiple interventions following an acute coronary syndrome event increase uptake into cardiac rehabilitation. Int J Cardiol 2021; 326:1-5. doi.org/10.1016/j.ijcard.2020.11.005.

9. Lo HC, Pazargadi A, Świątkiewicz I, Taub P. Secondary Prevention and cardiac rehabilitation. Springer, Cham, ASPC Manual of Preventive Cardiology 2021; 673-703. doi.org/ 10.1007/978-3-030-56279-3_27

10. Haq ZU, Khan D, Hisam A, Yousafzai YM, Hafeez S, Zulfiqar $F$, et al. Effectiveness of cardiac rehabilitation on health-related quality of life in patients with myocardial infarction in Pakistan. J Coll Physicians Surg Pak 2019; 29(9):803-09.

11. Beatty AL, Truong M, Schopfer DW, Shen H, Bachmann JM, Whooley MA. Geographic variation in cardiac rehabilitation participation in medicare and veterans affairs populations: An opportunity for improvement. Circulation 2018; 137(18):1899-908. doi.org/10.1161/CIRCULATIONAHA.117. 029471.

12. Jin K, Khonsari S, Gallagher R, Gallagher P, Clark AM, Freedman $B$, et al. Telehealth interventions for the secondary prevention of coronary heart disease: A systematic review and meta-analysis. Eur J Cardiovasc Nurs 2019; 18(4):260-71. doi.org/10.1177/1474515119826510

13. Hisam A, Haq ZU. Australian New Zealand Clinical Trials. [cited 2021]. Available from: http://www.anzctr.org.au/ Trial/Registration/TrialReview.aspx?id=375253\&isClinicalTrial=Falses

14. Hisam A, Haq ZU, Khan Z, Doherty P, Pell J. Mobile health augmented cardiac rehabilitation (MCard) in post-acute coronary syndrome patients: A randomised controlled trial protocol. PakJ Med Sci 2021; 37(3): 890-96. doi: 10.12669/ pjms.37.3.3664.

15. Sember V, Meh K, Sorić M, Starc G, Rocha P, Jurak G. Validity and reliability of international physical activity questionnaires for adults across EU countries: Systematic review and meta-analysis. Int J Environ Res Public Health 2020; 17(19):7161. doi.org/10.3390/ijerph17197161.

16. Vadiveloo M, Lichtenstein AH, Anderson C, Asprey K, Foraker R, Griggs $\mathrm{S}$, et al. American heart association council on lifestyle and cardiometabolic health; Council on arteriosclerosis, thrombosis and vascular biology; council on cardiovascular and stroke nursing; council on clinical cardiology; and stroke council. Rapid diet assessment screening tools for cardiovascular disease risk reduction across healthcare settings: A scientific statement from the American heart association. circ cardiovasc qual outcomes 2020; 13(9):e000094. doi.org/10.1161/HCQ.000000000 0000094

17. Reid R, Morrin L, Beaton L, Papadakis S, Tulloch H, D'Angelo MS, et al. The cardio fit expert system prescribes and tracks physical activity in patients with coronary artery disease (cad): Results from a randomised controlled trial. J Cardiopulm Rehabil Prev 2008; 28(5):335. doi: 10.1097/01. HCR.0000336147.24935.21.

18. Candelaria D, Randall S, Ladak L, Gallagher R. Health-related quality of life and exercise-based cardiac rehabilitation in contemporary acute coronary syndrome patients: A systematic review and meta-analysis. Qual Life Res 2019; 29:579-92. doi.org/10.1007/s11136-019-02338-y.

19. Francis $T$, Kabboul N, Rac V, Mitsakakis N, Pechlivanoglou P, Bielecki J, et al. The effect of cardiac rehabilitation on health-related quality of life in patients with coronary artery disease: A meta-analysis. Can J Cardiol 2019; 35(3): 352-64. doi.org/10.1016/j.cjca.2018.11.013.

20. Zhong VW, Van Horn L, Greenland P, Carnethon MR, Ning H, Wilkins JT, et al. Associations of processed meat, unprocessed red meat, poultry, or a fish intake with incident cardiovascular disease and all-cause mortality. JAMA Intern. Med 2020; 180(4):503-12. doi:10.1001/ jamainternmed. 2019.6969.

21. La Torre G, Cocchiara R, Sordo EL, Chiarini M, Siliquini R, Firenze $A$, et al. Counseling intervention improves the quality of life in patients with pre-existing acute myocardial infarction (AMI) or chronic obstructive pulmonary disease (COPD): A pilot study. J Prev Med Hyg 2018; 59(2): E153-E8.

22. Klimis $H$, Marschner $S$, Von Huben A, Thiagalingam A, Chow CK. Predictors of smoking cessation in a lifestyle-focused text-message support programme delivered to people with coronary heart disease: An analysis From the tobacco exercise and diet messages (TEXT ME) randomised clinical trial. Tobacco use insights 2020; 13:1179173X20901486. doi.org/10.1177/1179173X20901486

23. Sadeq AS, Elnour AA, Hamrouni AM, Baraka MA, Al Meslamani AZ, Adel A, et al. Development of a multivariable model to predict medication non-adherence risk factors acute coronary syndrome. J Pharm Health Serv Res 2021; 12(2):159-65. doi.org/10.1093/jphsr/rmab005

24. Abreu D, Sousa P, Matias-Dias C, Pinto FJ. Cardiovascular disease and high blood pressure trend analyses from 2002 to 2016: After implementing a salt reduction strategy. BMC Public Health 2018; 18(1):1-9. doi.org/10.1186/ s12889018-5634.

25. Sławska A, Siudak Z. Nurse-managed education: the effectiveness of secondary prevention after acute coronary syndromes and the prevalence and predictors of dropout from a cardiac rehabilitation programme. Postepy Kardiol Interwencyjnej 2021; 17(1):46-53. doi: 10.5114/aic.2021. 104767. 